\title{
Stochastic modeling of hot weather spells and their characteristics
}

\author{
M. N. Khaliq ${ }^{1, *}$, T. B. M. J. Ouarda ${ }^{2}$, P. Gachon' ${ }^{1}$, L. Sushama ${ }^{3}$ \\ ${ }^{1}$ Adaptation and Impacts Research Section, Atmospheric Science and Technology Directorate, Environment Canada, \\ Place Bonaventure, Northeast Tower, 800 Gauchetiere Street West, Office 7810, Montreal, Quebec H5A 1L9, Canada \\ ${ }^{2}$ INRS-ETE, University of Quebec, 490 de la Couronne, Quebec City, Quebec G1K 9A9, Canada \\ ${ }^{3}$ Centre ESCER, University of Quebec at Montreal, Quebec H3C 3P8, Canada
}

\begin{abstract}
Stochastic characterization of hot weather events (HWEs) is useful for developing probabilistic climate change information. We assumed that simultaneous exceedances of daily minimum and maximum temperatures (i.e. $T_{\min }$ and $T_{\max }$, respectively), above the selected thresholds for these temperatures, form alternating sequences of 'hot weather' and 'non-hot weather' spells. This behavior is stochastically characterized by a generalization of the compound Poisson process. The generalization is achieved by randomizing the mean rate of occurrence of the original Poisson process with the gamma distribution. The resulting compound Poisson process gamma (CPPG) model consists of 2 basic components: one relates to the occurrence of HWEs and the other to their durations. Detailed validation of the CPPG model is presented for HWEs derived from homogenized $T_{\min }$ and $T_{\max }$ observations from McTavish station (located in the centre of Montreal, southern Quebec, Canada) for the June-August hot summer season over the period 1941-2000. Results of the study suggest that the CPPG model can adequately describe occurrences of HWEs and the distributions of their durations (including distributions of extreme durations), as well as the distributions of the number of hot days, when assessed on the basis of the Kolmogorov-Smirnov and chi-squared goodness-of-fit tests. A nonstationary framework is introduced and applied to 2 contrasting sets of non-stationary observations of HWEs from Les Cedres and La Tuque (southern Quebec) for the 1941-2000 period. The results of the non-stationary modeling approach demonstrate that the developed methodology is generally applicable and could be useful in developing probabilistic information about various characteristics of hot weather climate for a particular location or a larger spatial domain.
\end{abstract}

KEY WORDS: Climate change - Compound Poisson process · Hot weather spells $\cdot$ Logarithmic distribution · Negative binomial distribution · Poisson-gamma model

\section{INTRODUCTION}

Extreme hot weather and climate phenomena are areas of great research interest, since increases in their frequency and severity can cause considerable damage to ecosystems and human society. The focus of the present study is on stochastic characterization of hot weather spells in southern Quebec, where most of the population of the province is concentrated and where high daily maximum temperatures, reaching $>30^{\circ} \mathrm{C}$ over various durations, during the summer season have occasionally been experienced (Khaliq et al. 2005). Extended episodes of such high temperatures and increases in their rate of occurrence under the anticipated climate change scenarios could considerably impact public health and socio-economic sectors.

Episodes of hot weather persisting over a certain number of days are termed hot weather events (HWEs) in the present study. Thus, in general, an HWE involves the occurrence of consecutive days of high temperatures, along with high levels of relative humidity. The high temperatures could be in the form of either daily maximum temperature (henceforth, 
denoted $T_{\max }$ ) or daily minimum temperature (henceforth, denoted $T_{\min }$ ) or the 2 together. There is no unified definition of an HWE, and its choice depends upon a number of factors, e.g. the type and length of the available data, the chosen sector impacted by HWEs (i.e. public health, agriculture, wildlife, socioeconomics, etc.), the time of year, local exacerbating forcing factors, etc. We define an HWE as an extreme climate event when-over a specific period-both $T_{\min }$ and $T_{\max }$ values are above relatively high chosen thresholds (see Section 3). These types of hot weather spells are supposedly more severe, in relation to their impact on public health and socio-economic sectors, than spells defined on the basis of $T_{\min }$ or $T_{\max }$ alone, such as in Katsoulis \& Hatzianastassiou (2005), Khaliq et al. (2005) and Furrer et al. (2010). As in Khaliq et al. (2006a, 2007), homogenized $T_{\min }$ and $T_{\max }$ observations for the June-August hot summer season are employed to derive HWEs. The effect of relative humidity, for which homogenized observations are not yet available, is not taken into account. However, inclusion of a relative humidity factor in the definition of an HWE would certainly be useful, since HWEs are likely more detrimental to humans under conditions of increased atmospheric moisture.

Following the above definition of an HWE, simultaneous observations of $T_{\min }$ and $T_{\max }$ would result in alternate sequences of 'hot weather' and 'non-hot weather' spells. In a very general manner, these sequences can be stochastically modeled using point process approaches (Cox \& Isham 1980, Guttorp 1995). In these approaches, the primary mechanism of event occurrences is usually assumed to follow a Poisson process, which is compounded with other secondary processes to formulate compound Poisson processes. Various forms of the compound Poisson process have been successfully used for modeling hydro-climatological data (e.g. rainfall, RodriguezIturbe et al. 1987; drought, Zelenhasić \& Salvai 1987, Abaurrea \& Cebrián 2002; wind storms, Rootzén \& Tajvidi 1997; hurricanes, Elsner \& Bossak 2001, Katz 2002; very cold days, Prieto et al. 2004; hot spells, Furrer et al. 2010). However, in practice, one could encounter situations where the assumptions of the Poisson process (e.g. equality of mean and variance property) may not be satisfied or they may hold merely in a weak manner. In such situations, the original Poisson process needs to be modified, particularly to account for over-dispersion in the event occurrence process; this is also the case in the present study. The present study has 2 main objectives. (1) To develop a stochastic characterization of hot weather climate using the compound Poisson process gamma (CPPG) model - a generalization of the Poisson process that reduces to the original form as a limiting case; gamma is the distribution of the rate parameter of the Poisson process. (2) To develop a plausible non-stationary probabilistic framework for hot weather activity in southern Quebec using data from 3 selected locations (Montreal, Les Cedres and La Tuque) with quite contrasting features of HWE occurrences and their characteristics.

\section{MODEL COMPONENTS}

In the present study, hot weather spells are characterized in terms of 4 components: (1) HWE occurrences, (2) HWE durations, (3) the number of hot days and (4) the longest duration of HWEs. The first 2 components (termed basic components) are explicitly modeled, while the distributions of the latter 2 are derived from those of the first 2. Other characteristics, like the intensity of HWEs (for example, represented in terms of the maximum temperature associated with a HWE) is not addressed explicitly in this study, but could be attempted in the future following the approaches described in Katsoulis \& Hatzianastassiou (2005) and Furrer et al. (2010) or their suitable variants in a multivariate framework. The 4 components, described below one at a time, correspond to the characteristics of HWEs for the June-August summer season.

\subsection{HWE occurrences}

It is assumed that the occurrence of HWEs follows a Poisson process $\{N\}$, denoting the number of events occurring within the time interval $(0, t)$, i.e. within the June-August (JJA) summer season of each year. The number of events in any interval of time is independent of the number of events in any other nonoverlapping interval of time. The random variable $N$ has a Poisson distribution with a mean rate $\lambda$. That is, the probability $P$ of $n$ events occurring within the interval $(0, t)$ can be expressed as:

$$
P(N=n)=\mathrm{e}^{-\lambda}(\lambda)^{n} / n !, \quad n=0,1,2, \ldots
$$

The mean $\mu_{N}$ and variance $\sigma_{N}^{2}$ of $N$ are equal and are given by $\mu_{N}=\sigma_{N}^{2}=\lambda$. In particular, for the case of rare events (i.e. the HWEs corresponding to relatively high values of $T_{\min }$ and $T_{\max }$ thresholds; see Section 3.2), the requirement of the equality of the mean and variance property of the Poisson distribution is not satisfied and the event occurrence process results in over-dispersion, i.e. $\sigma_{N}^{2}$ is significantly greater than $\mu_{N}$. To account for over-dispersion, generally the parameter of the Poisson distribution is assumed to follow a gamma distribution (Cameron \& Trivedi 1998, Medhi 2002), with the density function given by: 


$$
f_{\Lambda}(\lambda)=\frac{\beta^{\alpha}}{\Gamma(\alpha)} \lambda^{\alpha-1} \mathrm{e}^{-\beta \lambda}
$$

where $\alpha>0$ and $1 / \beta>0$ are, respectively, the shape and scale parameters and $\Gamma($.) is the gamma function. With this assumption, the distribution of $N$ becomes:

$$
\begin{aligned}
P(N=n) & =\int_{0}^{\infty} \mathrm{e}^{-\lambda} \frac{(\lambda)^{n}}{n !} \frac{\beta^{\alpha}}{\Gamma(\alpha)} \lambda^{\alpha-1} \mathrm{e}^{-\beta \lambda} \mathrm{d} \lambda \\
& =\left(\begin{array}{c}
\alpha+n-1 \\
n
\end{array}\right)\left[\frac{1}{1+\beta}\right]^{n}\left[\frac{\beta}{1+\beta}\right]^{\alpha}, \quad n=0,1,2, \ldots
\end{aligned}
$$

which is an extended negative binomial distribution with parameter $p=\beta /(1+\beta)$ and $r=\alpha$ in the form of commonly used notation. This formulation is known as a Poisson-gamma or Poisson mixture model in the statistical literature (e.g. Skinner 1992, Cameron \& Trivedi 1998). For the Poisson-gamma model, $\mu_{N}=$ $r(1-p) / p$ and $\sigma_{N}^{2}=r(1-p) / p^{2}$. Thus, over-dispersed cases can be easily modeled by estimating the parameters $p$ and $r$ using either the method of maximum likelihood or the method of moments for which the relationships for both the mean and variance need to be employed. An intuitively appealing explanation for the use of the Poisson-gamma model for HWE occurrences could be that: (1) it accommodates any random effect or heterogeneity (e.g. due to a seasonality effect on the rate of occurrence of HWEs), and (2) it reduces to the original Poisson process model as a limiting case, i.e. when the parameter $p$ approaches unity.

\subsection{Durations of HWEs}

Let $X_{i}>0$ denote the duration of HWE associated with the $i$ th occurrence, $i=1,2,3, \ldots$, within the JJA summer season of each year and assume that the $X_{i}$ s are independent and identically distributed. Let the common cumulative distribution function be denoted by $F_{X}(x)=P(X \leq X)$, with mean $\mu_{\mathrm{X}}$ and variance $\sigma_{N^{\prime}}^{2}$ and further, assume that the duration process $\{X\}$ is statistically independent of the occurrence process $\{N\}$. The joint process, consisting of $\{X\}$ in combination with $\{N\}$, is referred to as the compound Poisson process or a 'marked' Poisson process, i.e. the duration associated with an event occurrence is viewed as a mark; for additional explanations, see Cox \& Isham (1980) and Guttorp (1995). The randomized compound Poisson process could be viewed as an extension or a variant of the basic compound Poisson process. In practice $F_{X}(x)$ would be some positively skewed distribution function, such as the exponential, gamma, or lognormal from continuous distribution families and the truncated geometric, negative binomial, or logarithmic from discrete distribution families.
During a preliminary analysis, 3 single-parameter distributions (exponential, logarithmic and truncated geometric) were considered to identify strong candidate distributions for modeling HWE durations. The exponential is a continuous distribution, while the other 2 are discrete. The exponential distribution is considered because of its extensive use in climatology and hydrology. Based on the Kolmogorov-Smirnov and chi-squared goodness-of-fit tests (Snedecor \& Cochran 1980), the logarithmic and truncated geometric distributions were found to be adequate for modeling HWE durations, compared to the exponential distribution. Though the latter is the continuous counterpart of the geometric distribution, it was not able to estimate frequencies of $1 \mathrm{~d}$ duration satisfactoryly. Based on the results of this preliminary investigation, the logarithmic distribution was selected in the present study for further theoretical development. The distribution function of the logarithmic distribution, with the mean $\mu_{X}=-b /(a \log a)$ and variance $\left(\mu_{X} a^{-1}-\mu_{X}^{2}\right)$, is given below (Kendall \& Stuart 1977):

$$
P(X=x)=\frac{-b^{x}}{x \log a}, \quad 0<a<1 \text { and } b=1-a
$$

where $a$ is the parameter of the distribution. The exponential distribution is also studied to compare its results with those of the logarithmic distribution; however, only selected results are presented for this distribution.

\subsection{Longest duration of HWEs}

Under the assumption of the compound Poisson process, the distribution function of the longest duration of HWEs $\left(X^{*}\right)$ can be derived from the following relationship:

$$
F_{X^{*}}\left(X^{*}\right)=P\left(N_{0}^{t}\right)+\sum_{k=1}^{\infty}\left\{\left[F_{X}\left(X^{*}\right)\right]^{k} P\left(N_{k}^{t}\right)\right\}
$$

where the expression $P\left(N_{k}^{t}\right)$ represents the probability of $k$ occurrences of HWEs in the interval $(0, t)$ (i.e. JJA period) and $F_{X}($.$) is the distribution function of all$ durations of HWEs. In general, $F_{X} \cdot\left(X^{*}\right)$ has to be approximated by numerical means. In the case of the exponential distribution for HWE durations and a Poisson distribution for $P\left(N_{k}^{t}\right), F_{X^{*}}\left(X^{*}\right)$ results in a closed form relationship, which is equivalent to the Gumbel distribution of the maximum of a sample of observations (for additional details, see Zelenhasić \& Salvai 1987). In the case of the Poisson distribution for $P\left(N_{k}^{t}\right), F_{X^{*}}\left(X^{*}\right)$ can also be derived in terms of the survival function for the spell length following Reiss \& Thomas (2007, p. 29). 


\subsection{Number of hot days}

The number of hot days refers to the number of days with $T_{\min }$ and $T_{\max }$ simultaneously above specified thresholds (which are discussed later in Sections 3 \& 4) observed during the JJA summer season of each year. Under the assumption of the CPPG model for occurrence of HWEs and their durations, the number of hot days $(M)$ can be expressed as the sum:

$$
M=X_{1}+X_{2}+\ldots+X_{i}+\ldots+X_{N=n}
$$

(conditional on $N \geq 1$; otherwise, $M=0$ ). This representation is termed a random sum, because the number of terms $N$ is not fixed a priori. The mean and variance of the random sum $M$ can be expressed in terms of the parameters of the 2 component processes through conditioning on the number of events $N$. Making use of the relationship between the unconditional mean and the conditional mean, the mean of $M$ (denoted $\mu_{M}$ ) is given by:

$$
\begin{aligned}
\mu_{M} & =E\{E[M \mid N]\}=E[N] E[X]=\mu_{N} \mu_{X} \\
& =-r(1-p)(1-a) /[p(b \log b)]
\end{aligned}
$$

(see Chapter 12 in Feller 1968) where $E$ is the expectation operator. Similarly, making use of the relationship between the unconditional variance and the conditional means and conditional variances, as well as of the expressions for the mean and variance of the Poisson-gamma model, the variance of $M$ (denoted $\sigma_{M}^{2}$ ) is given by:

$$
\begin{aligned}
\operatorname{Var}[M]=\sigma_{M}^{2} & =E\{\operatorname{Var}[M \mid N]\}+\operatorname{Var}\{E[M \mid N]\} \\
& =E[N] \operatorname{Var}[X]+\operatorname{Var}[N][E(X)]^{2} \\
& =\frac{-b r(1-p)}{p a(\log a)}\left[\frac{1}{a}+\frac{b}{a(\log a)}\right]+\frac{b^{2} r(1-p)}{p^{2} a^{2}(\log a)^{2}}
\end{aligned}
$$

Other approaches for the derivations of $\mu_{M}$ and $\sigma_{M}^{2}$ are given in Parzen (1964, p. 130-131) and Medhi (2002, p. 1-3, 176-177) using the moment and probability generating functions.

If $X$ is assumed to follow the discrete logarithmic distribution, as explained earlier (Section 2.2), then the probability distribution of $M, P(M=m)$ in the case of the basic compound Poisson process (i.e. when Eq. (1) is applicable), can be written as:

$$
P(M=m)=(-1)^{m} \frac{b^{m} \mathrm{e}^{-\lambda}}{m !} \sum_{n=0}^{m} S N F_{m}^{n}\left(\frac{\lambda}{\log a}\right)^{n}
$$

It should be noted that Eq. (9) is an alternate form of the extended negative binomial distribution. In the case of the CPPG model, this relationship becomes:

$$
P(M=m)=(-1)^{m} \frac{b^{m} p^{r}}{m ! \Gamma(r)} \sum_{n=0}^{m} S N F_{m}^{n}\left(\frac{q}{\log a}\right)^{n} \Gamma(r+n)
$$

Detailed derivations of the 2 above relationships are provided in the Appendix.

\section{A VALIDATION FOR OBSERVATIONS OF HOT WEATHER SPELLS}

In this section, detailed results and a discussion of an application of the CPPG model to observations of HWEs for Montreal, which is one of the 3 locations studied from southern Quebec, are presented. A description of the $T_{\min }$ and $T_{\max }$ data along with their thresholds and basic statistics of some characteristics of HWE observations are presented first, followed by the results of the application.

\section{1. $T_{\min }$ and $T_{\max }$ data, their thresholds and statistics of hot weather spells}

HWEs are derived from homogenized $T_{\min }$ and $T_{\max }$ observations recorded at McTavish station, located in the centre of Montreal, over the period 1941-2000. The homogenized observations have been developed by Vincent et al. $(2000,2002)$ for climate change studies in Canada. Since the focus of the study is on HWEs, only observations of the hot summer season (JJA) are considered for the analysis. For the 5 yr, 1992-1996, summer season $T_{\min }$ and $T_{\max }$ observations at McTavish station are either not available or sparsely observed, and therefore these years are excluded from the analysis. In the case of very few missing temperature values for the remaining years of the period of study, termination of an HWE or non-HWE is considered whenever a day with missing temperature value is encountered.

Four thresholds for the $T_{\min }$ (i.e. $u=18,19,20$ and $21^{\circ} \mathrm{C}$ ) and 6 thresholds for the $T_{\max }$ (i.e. $V=27,28,29$, 30,31 and $32^{\circ} \mathrm{C}$ ) are selected initially to derive HWEs for Montreal. These thresholds are selected from the upper quartile of the distributions of $T_{\min }$ and $T_{\max }$ after pooling the seasonal observations for the period of study. Clearly, the choice of these thresholds is arbitrary but reasonable in that it allows study of a wide range of hot weather spells of various characteristics and at the same time helps maintain their extreme character and severity through the threshold exceedance mechanism. For the convenience of presentation, each combination of $u$ and $v$ thresholds is referred to as $u: v$ in the current paper.

The next step is to test the autocorrelation and temporal structure of time series of various characteristics of HWEs (i.e. the number of occurrences $N$, duration $X$, number of hot days $M$ and longest duration $X^{*}$ ), because the results of the analyses may be affected by these factors. None of the time series, for any of the characteristics of HWEs, is found to be autocorrelated at the $5 \%$ level following the testing procedure for the first autocorrelation coefficient described in Anderson (1942), by using asymptotic confidence intervals (Box 
\& Jenkins 1970), or by using Spearman rank order correlation coefficients (Walpole \& Myers 1989). As for the temporal structures where various time series of HWE characteristics are concerned, none of them is found to be non-stationary (i.e. there is insufficient evidence in favor of temporal trends) using the Mann-Kendall (Kendall 1975) and Spearman rank correlation testing procedures. The independence assumption of the observations of inter-event waiting time (i.e. the time interval between the start of a HWE and the start of the following HWE) and the duration of HWEs is checked using the test for the first autocorrelation coefficient. All observed samples of inter-event waiting times and HWE durations are found independent at $5 \%$ significance level, except the sample of HWE durations corresponding to the $u: v=20: 30$ case, which is found to be independent at about the $7 \%$ level. In the present study, this sample of HWE durations is also assumed to be independent.

The basic statistics of $N$ (i.e. $\hat{\mu}_{N}$ and $\hat{\sigma}_{N}^{2}$ ), $X$ (i.e. $\hat{\mu}_{X}$ and $\hat{\sigma}_{X}^{2}$ ) and $M$ (i.e. $\hat{\mu}_{M}$ and $\hat{\sigma}_{M}^{2}$ ) are plotted in Fig. 1 for various $u: v$ cases. Such plots help one to visually identify the inter-relationships among moments of various characteristics. For example, it is clear from Fig. 1 that for a particular value of the threshold $u$, the mean is a decreasing function of the threshold $v$ (i.e. $\hat{\mu}_{N}, \hat{\mu}_{X}$ and $\hat{\mu}_{M}$ decrease systematically, in general, as $v$ increases), but not necessarily the variance. Whether the observed data support the equality of the mean and variance property of the Poisson distribution can be examined from the respective plots given in Fig. 1. In the case of the exponential distribution for durations of HWEs, $\sigma_{X}^{2}=\mu_{X}^{2}$, which does not seem to hold for the majority of the $u: v$ combinations. From the plots of $\hat{\mu}_{M}^{2}$ and $\hat{\sigma}_{M}^{2}$, it is obvious that a definite relationship exists between these moments, i.e. $\hat{\sigma}_{M}^{2}$ is some (say $h$ ) multiple of $\hat{\mu}_{M}^{2}$.

\subsection{Distribution of number of HWEs}

As explained earlier in Section 2, where applicable, it is assumed that the occurrence of HWEs follows a Poisson process or a generalization of the Poisson process (i.e. the Poisson-gamma model). The Poissongamma model is suitable in situations where noticeable departures from the assumption of the simple Poisson process are realizable. Therefore, it is necessary to check the suitability of the Poisson process first before using the Poisson-gamma model. This can be achieved by using the fundamental property of equality of the mean and variance of the Poisson distribution and testing it by calculating the chi-squared test statistic given in Johnson et al. (1992), i.e.:

$$
\chi^{2}=(j-1) \hat{\sigma}_{N}^{2} / \hat{\mu}_{N} \sim \chi_{j-1}^{2}
$$

where $\hat{\mu}_{N}$ and $\hat{\sigma}_{N}^{2}$, respectively, denote the sample mean and variance of the seasonal counts of HWEs, i.e. the number of HWEs that occurred during the JJA summer season, and $j$ being the number of years. The
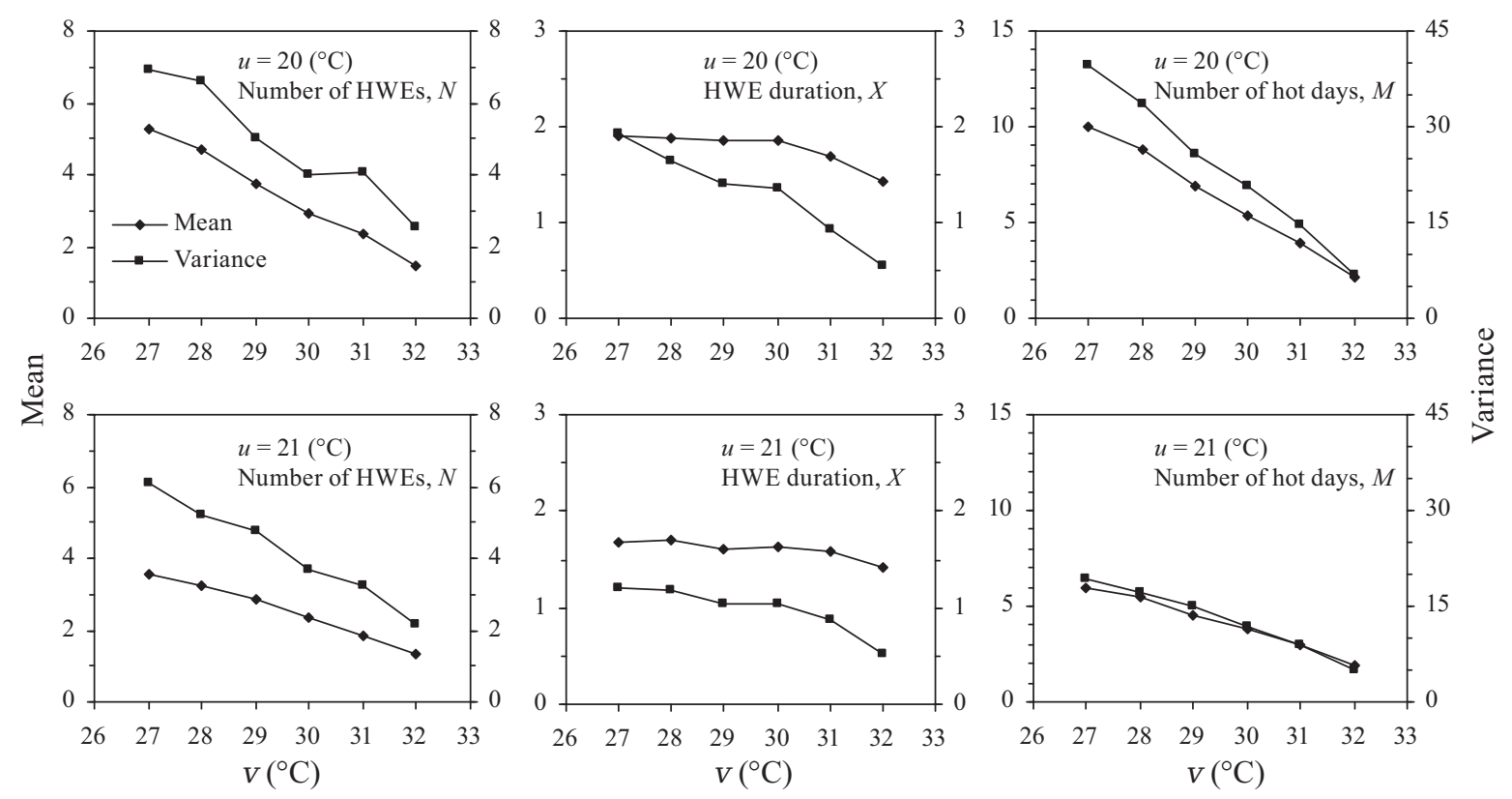

Fig. 1. Values of the mean and variance of number of occurrences $(N)$ and duration $(X)$ of hot weather events (HWEs) and the number of hot days $(M)$ corresponding to selected combinations of $T_{\min }(u)$ and $T_{\max }(v)$ thresholds for hot weather climate in Montreal 
calculated values of $\chi^{2}$ and corresponding $95 \%$ confidence intervals are shown in Fig. 2 for various combinations of $u$ and $v$ thresholds. It is obvious from this figure that HWE occurrences could be assumed to follow the Poisson process only for a certain number of combinations of $u$ and $v$ thresholds, because many calculated chi-squared values lie well outside the $95 \%$ confidence interval. Thus, there is inadequate statistical evidence to assume that HWEs corresponding to selected higher thresholds follow a Poisson process of event occurrences. To further confirm this result, 5000 bootstrap samples for each $u: v$ case, were generated and the $95 \%$ upper and lower confidence limits were noted for the $\chi^{2}$ test statistic given in Eq. (11). Almost the same results were found as those presented in Fig. 2 and discussed above (this section).

Also, the Kolmogorov-Smirnov and chi-squared goodness-of-fit tests resulted in the same conclusion as discussed in the previous paragraph. The Kolmogorov-Smirnov test requires the underlying distribution to be continuous, with no estimated parameters. However, it can be used for discrete distributions and when the parameters are estimated from observations, but it will provide only approximate results. In the latter case, the test is more conservative, i.e. if the critical value is exceeded by the test, then the hypothesis can be rejected with considerable confidence (Crutcher 1975, Von Storch \& Zwiers 1999). Based on the results presented above, it is logical to use the generalized form of the Poisson process (i.e. the Poisson-gamma model) to model occurrences of HWEs corresponding to higher thresholds.

In the remainder of the present paper, HWEs corresponding to only 6 combinations of relatively high values of $u$ and $v$ thresholds (i.e. $u=20$ and $21^{\circ} \mathrm{C}$ and $v=30,31$ and $32^{\circ} \mathrm{C}$ ) are considered for additional analyses. It is necessary to point out that the $u: v=$ 20:30 combination marginally satisfies the equality of mean and variance property of the Poisson distribution. Observed and modeled distribution functions of the number of HWEs for only 3 selected combi-

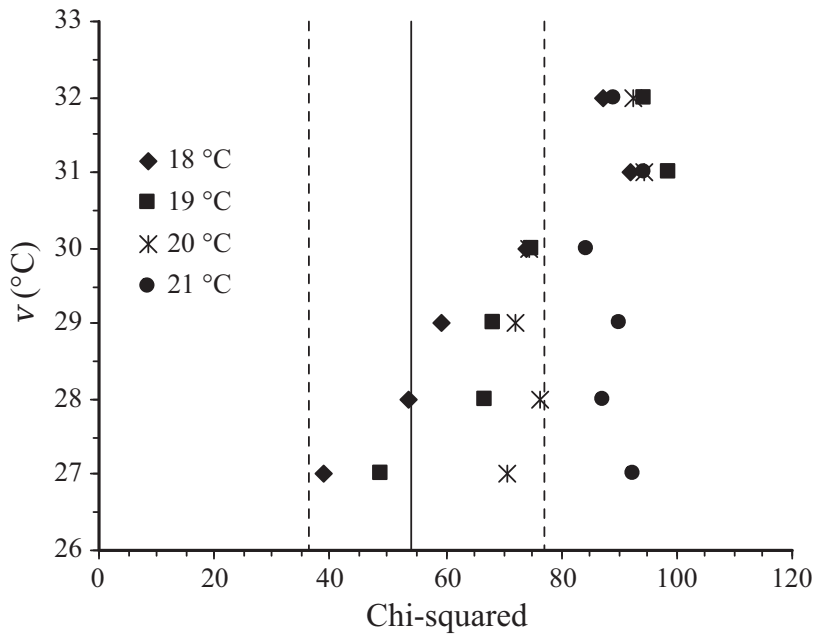

Fig. 2. Calculated chi-squared values and their 95\% confidence intervals (dotted lines) for selected combinations of $T_{\max }\left(v\right.$, shown on the $y$-axis) and $T_{\min }$ (symbols in the key) thresholds. Solid vertical line: equality of mean and variance property of the Poisson distribution

nations of $u$ and $v$ thresholds are shown in Fig. 3. This figure suggests that the Poisson-gamma model fits observed frequencies much better than the simple Poisson model, perhaps due to the reasons discussed in Section 2.1.

\subsection{Distribution of HWE durations}

Observed frequencies of HWE durations and the fitted exponential and logarithmic distributions, using the method of maximum likelihood, are shown in Fig. 4. The goodness-of-fit of the exponential and logarithmic distributions is assessed on the basis of the Kolmogorov-Smirnov and chi-squared goodness-of-fit tests. The Kolmogorov-Smirnov test rejects the fitting by the exponential distribution, but does not reject that by the logarithmic distribution at the $5 \%$ significance level for all $6 u: v$ cases. The chi-squared goodness-of-
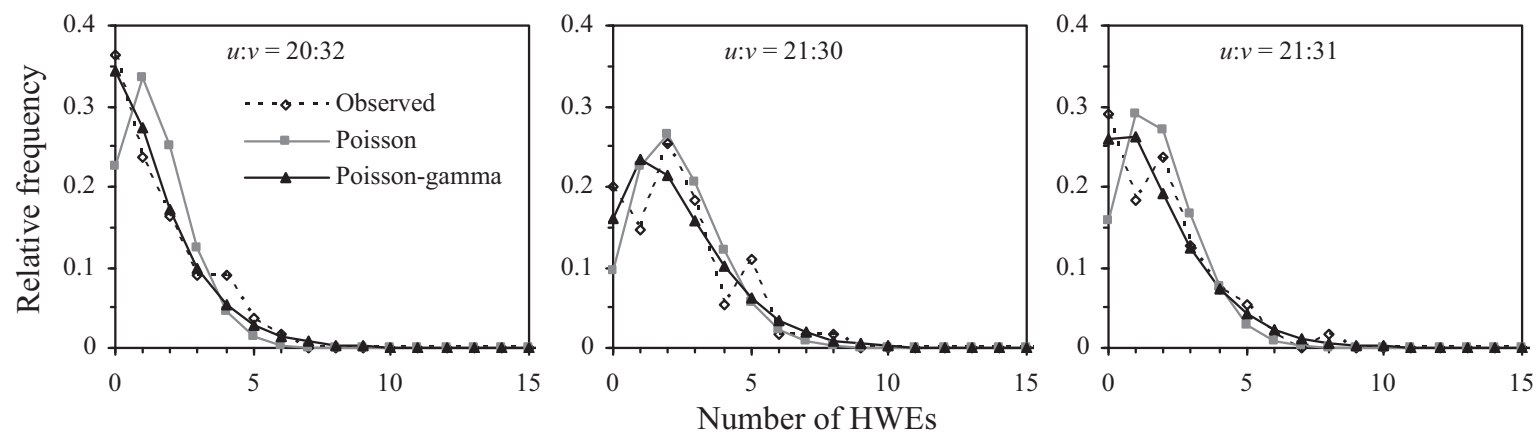

Fig. 3. Observed and modeled distributions of the number of hot weather events (HWEs) for 3 selected combinations of $T_{\min }(u)$ and $T_{\max }(\mathrm{V})$ thresholds (both in ${ }^{\circ} \mathrm{C}$ ) 
fit test is conducted by considering 5, 10 and 15 number of classes (each at an interval of $1 \mathrm{~d}$, with the first one representing $1 \mathrm{~d}$ duration); for this test, the numbers of rejected tests (at the $5 \%$ significance level) are 8 for the exponential distribution and just 1 for the logarithmic distribution, out of a total of 18 tests. Thus, the combined results of the 2 goodness-of-fit tests suggest that the logarithmic distribution describes the observed distributions of HWE durations better than the exponential distribution, which does not describe the observed frequencies of HWEs of $1 \mathrm{~d}$ duration well.

\subsection{Distribution of the longest duration of HWEs}

Observed and estimated distributions of the longest duration of HWEs, $X^{*}$, are shown in Fig. 5. It should be noted that the statistics of the observed distributions of $X^{*}$ are not used to estimate the theoretical distributions of $X^{*}$, because the latter are directly expressible in terms of the distributions of the 2 basic component processes of the CPPG model, i.e. the one relating to the occurrences of HWEs and the other to their durations. The fitting of the theoretical distributions to observations of $X^{*}$ is assessed on the basis of the Kolmogorov-Smirnov and chi-squared goodness-of-fit tests. The Kolmogorov-Smirnov test fails to reject fitting (at the $5 \%$ significance level) by the CPPG model with the logarithmic distribution for HWE durations for all the $u: v$ cases, but rejects fitting by the CPPG model with the exponential distribution for HWE durations for 3 (out of 6 ) $u: v$ cases. The chi-squared test is carried out by dividing the empirical distributions into 5, 8 and 10 class intervals, and the numbers of rejected tests, at the $5 \%$ level, are found to be 2 in the case of the exponential distribution and 0 in the case of the logarithmic distribution. Also, visual inspection of Fig. 5 indicates that the CPPG model with the logarithmic distribution for HWE durations estimates distributions of the longest duration of HWEs much better than the CPPG model with the exponential distribution for HWE durations.

The results presented and discussed so far suggest that the CPPG model with the logarithmic distribution for HWE durations describes occurrences of HWEs and their durations - as well as distributions of the longest duration of HWEs - better than the CPPG model with the exponential distribution for HWE durations. Therefore, only the former construction is considered for the additional analyses presented in the current paper.

\subsection{Distribution of number of hot days}

Observed and estimated distributions of number of hot days $(M)$ are shown in Fig. 6 for $6 u: V$ cases. It should be noted that none of the parameters of the theoretical distributions of $M$ are estimated from empirical observations of $M$, because parameters of these theoretical distributions are directly expressible in terms of the parameters of the 2 component processes of the CPPG model (i.e. $\{N\}$ and $\{X\}-$ see Section 2). The fitting of the theoretical distributions is assessed on the basis of the Kolmogorov-Smirnov and chi-squared
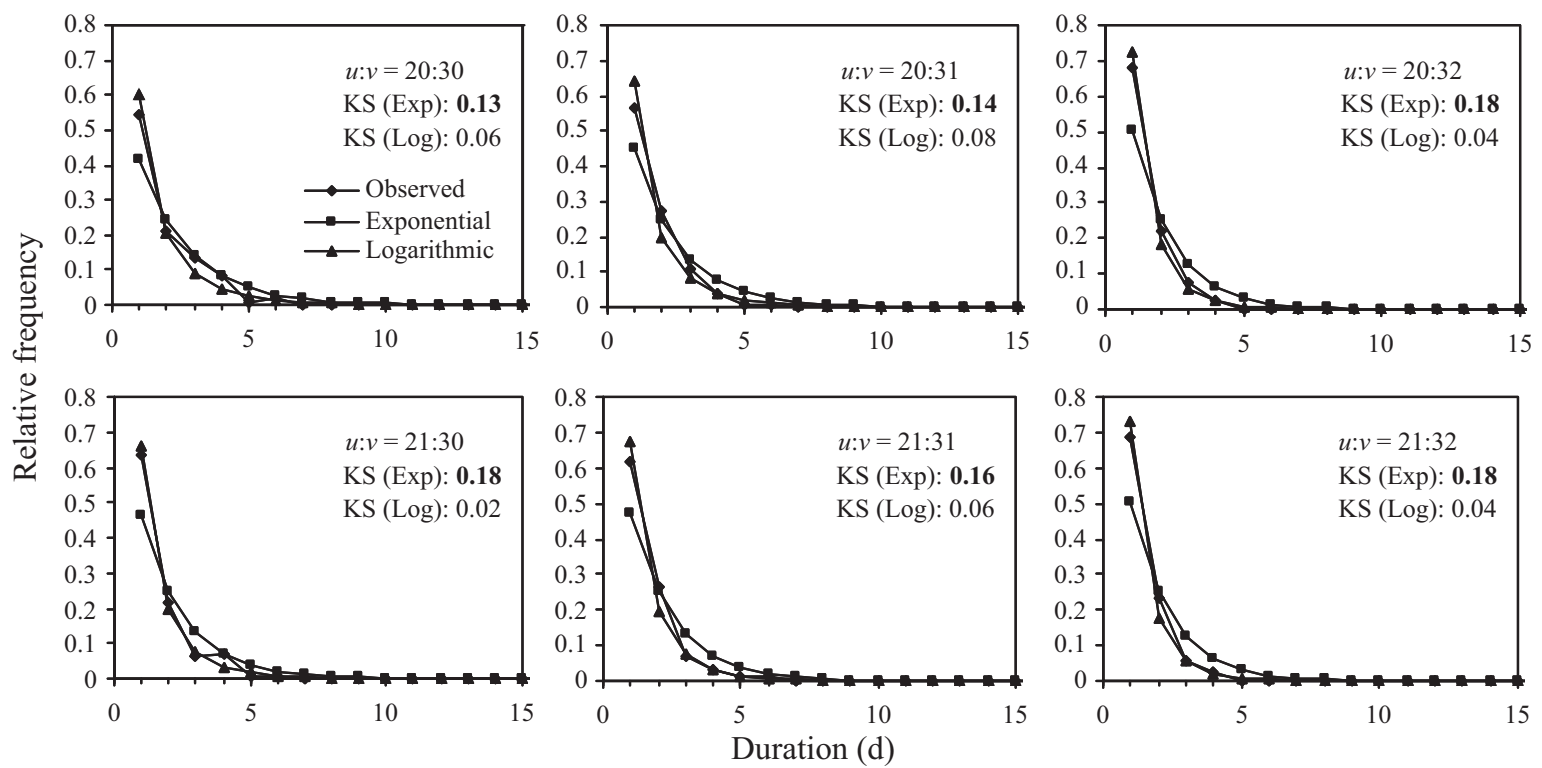

Fig. 4. Observed and estimated frequencies - with the exponential (Exp) and logarithmic (Log) distributions - of hot weather event durations for 6 selected combinations of $T_{\min }(u)$ and $T_{\max }(v)$ thresholds (both in ${ }^{\circ} \mathrm{C}$ ). The values of the KolmogorovSmirnov test statistics [KS (Exp) and KS (Log)] are listed in each panel. Bold: lack-of-fit (5\% level) 
goodness-of-fit tests. Neither the Kolmogorov-Smirnov nor the chi-squared (applied with 10, 15 and 20 class intervals) test rejects the fitting at the $5 \%$ significance level for all the $u: v$ cases. Although the mean of $M\left(\mu_{M}\right)$ is exactly preserved by the CPPG model through its structure (see Fig. 7), an alternate method of goodnessof-fit is to check how well the variance of $M\left(\sigma_{M}^{2}\right)$ is estimated. For this purpose, both the observed and estimated standard deviations of $M$ along with the $95 \%$ confidence intervals are plotted in Fig. 7 for all $u: v$ cases. It is interesting to note that the CPPG model does a good job in estimating $\sigma_{M}^{2}$.

\section{A NON-STATIONARY FRAMEWORK FOR CHARACTERISTICS OF HOT WEATHER SPELLS}

The temperature regimes are likely to change as a result of anticipated climate change, as are the frequency and severity of extreme events such as hot weather spells (IPCC 2002). Therefore, a non-stationary framework is needed to characterize HWEs and to develop probabilistic climate change information about hot weather climate. It is shown in Section 2 that distributions of the number of hot days are explicitly determined by the parameters of the 2 component processes, i.e. the HWE occurrence and duration processes. In other words, the moments of the distribution of the number of hot days are expressible in terms of the moments of the 2 component processes. Thus, any linear or non-linear temporal change in the moments of the distributions of the number of HWEs and durations of HWEs will be directly reflected in the moments of the distribution of the number of hot days. That is, either the frequency of occurrence or the duration of HWEs or the 2 together could change in response to changes in the temperature regime. Considering a time-dependant change, both $\mu_{N}$ and $\sigma_{N}^{2}$ can be represented as a function of time, i.e. $\mu_{N}(t)$ and $\sigma_{N}^{2}(t)$. Similar time-dependencies can also be introduced in $\mu_{X}$ and $\sigma_{X}^{2}$ as $\mu_{X}(t)$ and $\sigma_{X}(t)$. Folowing these timedependencies, the parameters of the distributions of $N$ (and $X$ ) can be expressed in terms of $\mu_{N}(t)$ and $\sigma_{N}^{2}(t)$ $\left(\mu_{X}(t)\right.$ and $\left.\sigma_{X}^{2}(t)\right)$, which, in turn, along with the parameters of the assumed forms of the time-dependencies, can be estimated by maximum-likelihood techniques using observed samples. The advantage of the maximumlikelihood technique is its asymptotic efficiency as compared with the method of moments, which is sensitive to extremely large observations (so-called outliers). A similar approach has been adopted for developing nonstationary flood-frequency relationships; see, for example, Strupczewski et al. (2001), Katz et al. (2002) and Khaliq et al. (2006b).

It is shown in Section 3 that there is inadequate statistical evidence in favor of non-stationarity for the hot weather climate in Montreal; therefore, the application of the non-stationary framework is demonstrated using hot weather observations from La Tuque and Les Cedres (southern Quebec). La Tuque is located north of Montreal at $47.40^{\circ} \mathrm{N}$ latitude and $72.78^{\circ} \mathrm{W}$ longitude, while Les Cedres is located west of Montreal at $45.30^{\circ} \mathrm{N}$ latitude and $74.05^{\circ} \mathrm{W}$ longitude. The proposed modeling approach was also found suitable for characterizing HWEs at these 2 locations; however, detailed evaluation results are omitted due to space constraints. Compared to Montreal, for which a range of plausibly
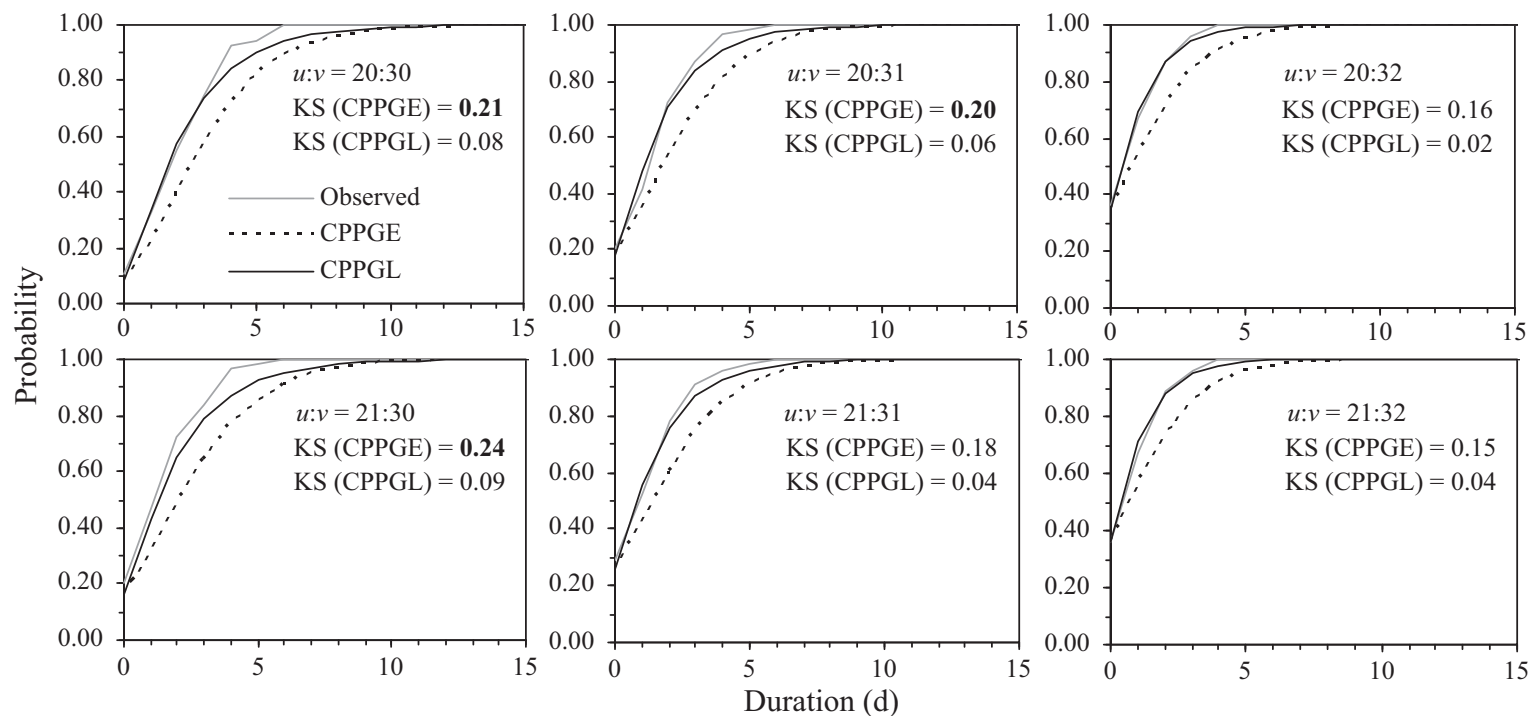

Fig. 5. Observed and estimated cumulative distribution functions of the longest duration of hot weather events (HWEs), for 6 selected combinations of $T_{\min }(u)$ and $T_{\max }(v)$ thresholds (both in ${ }^{\circ} \mathrm{C}$ ), for the compound Poisson process gamma (CPPG) model with exponential and logarithmic distributions for HWE durations (CPPGE and CPPGL). The values of Kolmogorov-Smirnov test statistics (KS) are listed in each panel. Bold: lack-of-fit (5\% level) 

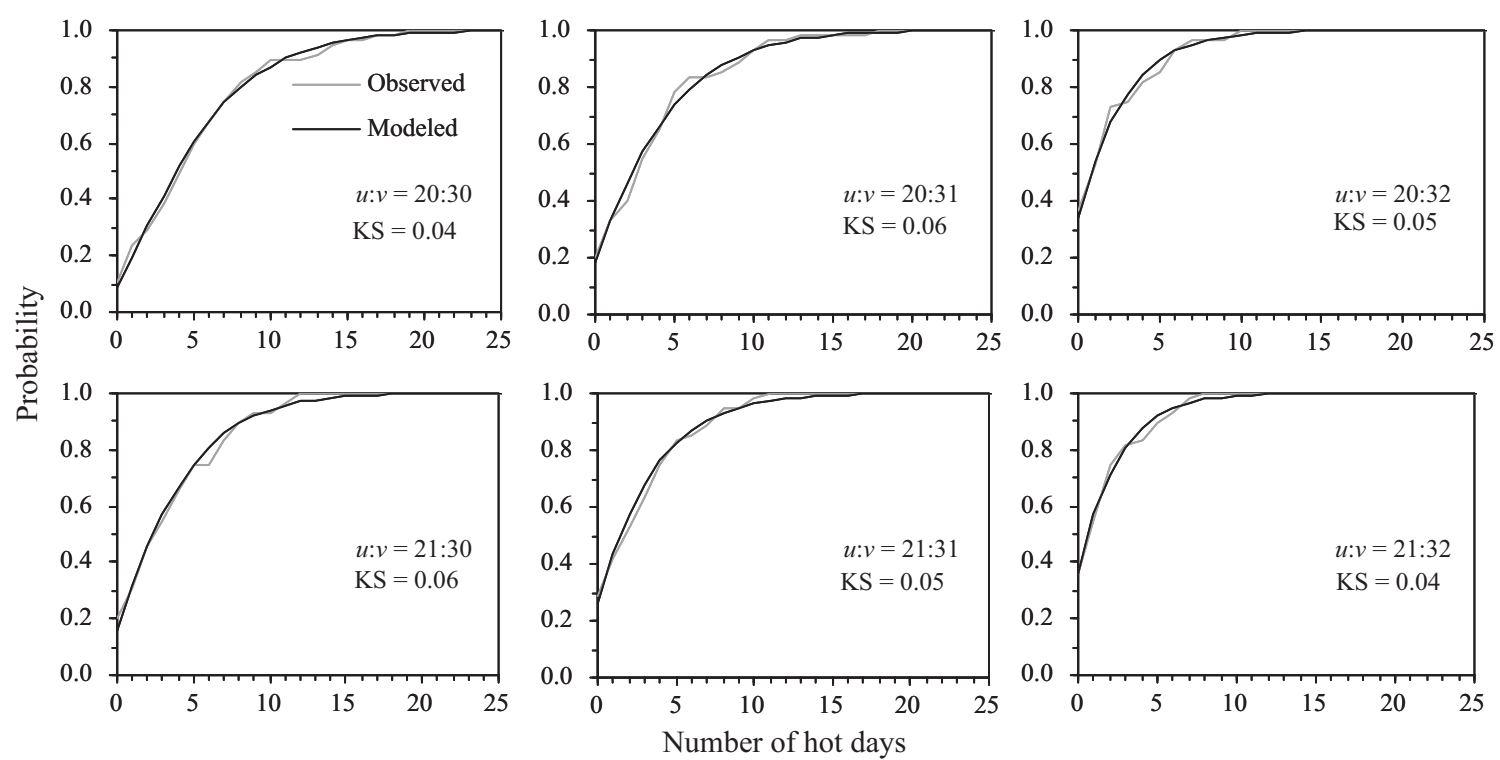

Fig. 6. Observed and modeled distribution functions of the number of hot days $(M)$ for 6 different combinations of $T_{\min }(u)$ and $T_{\max }(v)$ thresholds (both in ${ }^{\circ} \mathrm{C}$ ). The values of Kolmogorov-Smirnov test statistics (KS) are listed in each panel, and none of them suggest lack-of-fit at the $5 \%$ level

selected subjective thresholds is employed, percentilebased thresholds are used to derive HWEs for these 2 locations. Two typical time series of seasonal counts of HWEs, which exhibit contrasting non-stationarity, are selected and shown in Fig. 8. Both the Mann-Kendall and Spearman rank correlation tests detect significant time trends at the $4 \%$ level for counts of HWEs for both locations. For the time series of the number of hot days, the Mann-Kendall test detects a significant time trend
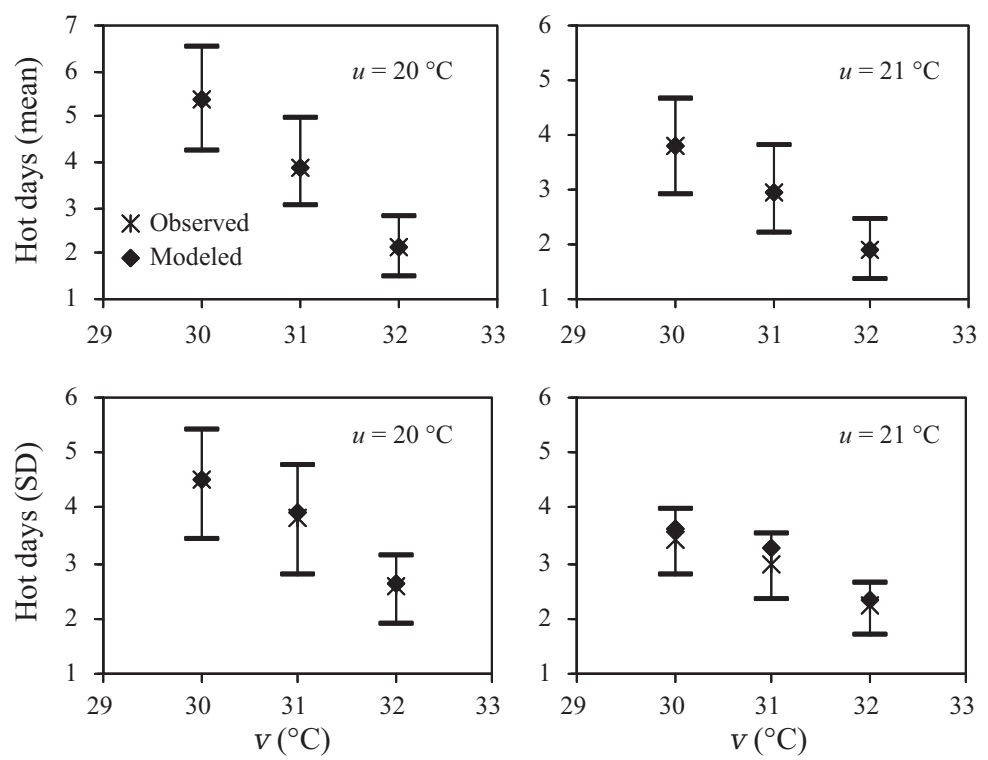

Fig. 7. Observed and modeled values of the mean (upper panels) and standard deviation (SD, lower panels) of the number of hot days corresponding to 6 different combinations of $T_{\min }(u)$ and $T_{\max }(v)$ thresholds. Error bars: $95 \% \mathrm{CI}$, obtained using 5000 bootstrap samples at the $3 \%$ level for Les Cedres and at the $9 \%$ level for La Tuque, and the Spearman rank correlation test detects a significant trend at the $4 \%$ level for Les Cedres and at the $13 \%$ level for La Tuque. It is important to mention here that the existence of non-stationarity is also dependent on the choice of the thresholds used to derive HWEs for these 2 locations. None of the time series of HWE characteristics at either location is found to be autocorrelated.

To model HWE occurrences at La Tuque and Les Cedres, 5 trend models are considered, i.e. (1) a linear trend in $\mu_{N}$ of the form $\mu_{N}(t)=a_{\mu_{N}}+b_{\mu_{N}} t$, (2) a linear trend in $\sigma_{N}^{2}$ of the form $\sigma_{N}^{2}(t)=a_{\sigma_{N}}+b_{\sigma_{N}} t$, (3) a joint linear trend in both $\mu_{N}$ and $\sigma_{N}^{2}$, (4) a non-linear trend in $\mu_{N}$ of the form $\mu_{N}(t)=a_{\mu_{N}}+b_{\mu_{N}} t+C_{\sigma_{N}} t^{2}$ and (5) a non-linear trend in $\sigma_{N}^{2}$ similar to the form for $\mu_{N}$, where $a, b$ and $c$ are parameters of the trend model and $t$ is the time index. It is also necessary to mention here that the simple Poisson process is adequate to model HWE occurrences for La Tuque on the basis of the chi-squared test given in Eq. (11). Therefore, HWE occurrences are modeled with the Poisson-gamma model for Les Cedres and with the original Poisson process for La Tuque. The parameters of Eqs. (1) and (3) were replaced with the trend model parameters, which, in turn, were estimated by maximizing the likelihood function using the non-linear optimization algorithm from the R statistical computing software (www.r-project.org/). In order to 
choose from among the stationary and non-stationary models, the Akaike information criterion (AIC; Akaike 1974) is used, which is defined as AIC $=-2 \ln (\mathrm{ML})+2 k$, where ML is the maximum likelihood and $k$ is the number of estimated parameters. From the set of various competing models, the one with the smallest value of the AIC is the preferred model. Based on the AIC, the preferred non-stationary model is the one with the linear trend in the mean for both locations. A similar exercise did not result in any form of the nonstationary model for HWE durations; hence, this characteristic can be assumed stationary. Following the selected non-stationary model, temporal evolution of exceedance probabilities of the longest HWE durations and those of the number of hot days were developed, and results for a few selected cases are shown in Fig. 9 for the former characteristic and in Fig. 10 for the latter characteristic.

Both Figs. $9 \& 10$ show that the compound Poisson process, in its basic and generalized form, with the assumed trend model, captures well the non-stationarity present in occurrences of HWEs and its effect on the longest duration of HWEs and the number of hot days. Thus, it can be concluded that the proposed nonstationary modeling framework can be a useful tool for developing probabilistic information about hot weather climate in southern Quebec.
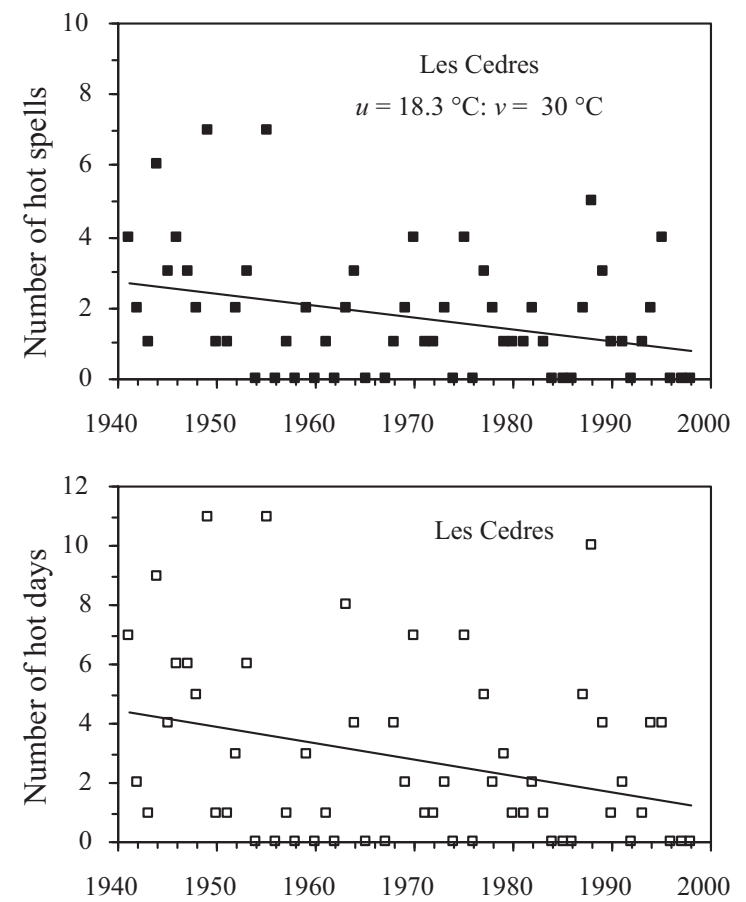

\section{SUMMARY AND CONCLUSIONS}

In the present paper, a stochastic point process approach is proposed for modeling the occurrence of HWEs and their characteristics. A generalized compound Poisson process (i.e. CPPG model) is formulated to model various characteristics of HWEs (i.e. frequency of occurrence and duration of HWEs, including distributions of the longest duration and number of hot days) observed during the June-August summer period at 3 different locations in southern Quebec that exhibit quite contrasting features. The choice of the stochastic point process approach rests completely on its ability to adequately describe various component processes, with little physical justification. The generalization of the compound Poisson process is achieved by randomizing the mean rate of occurrence parameter using a gamma distribution to model over-dispersion in the occurrence process. The use of the gamma distribution is natural in that the resulting generalized model reduces to the original model as a limiting case. The single-parameter exponential and logarithmic distributions are analyzed to model HWE durations because of their simplicity. Other distributions with $>1$ parameter are not investigated in the present study, and could be considered in future studies. HWEs are defined using the notion of threshold exceedances on
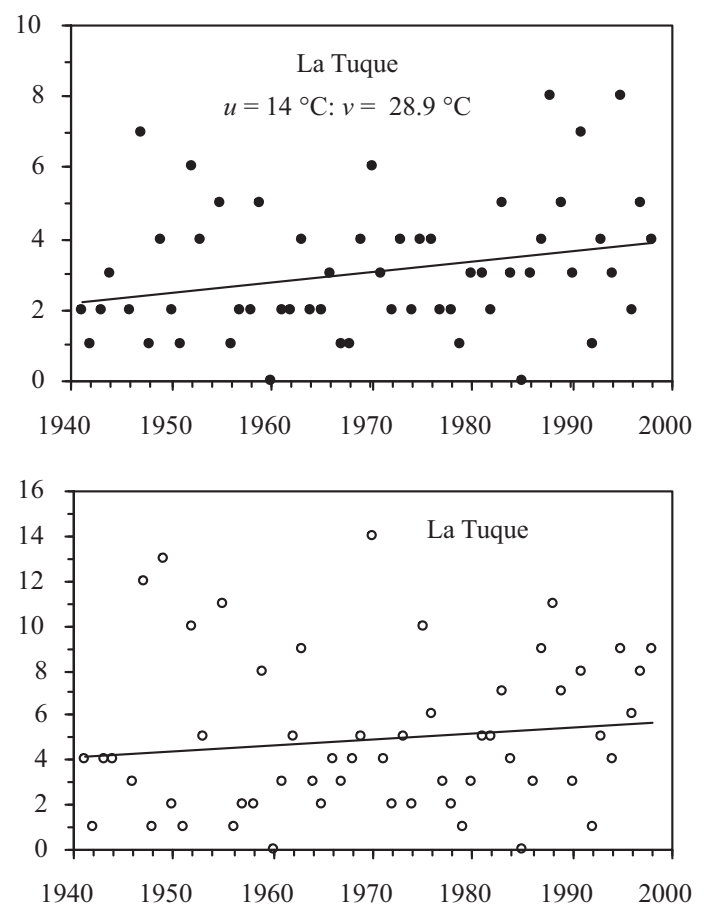

Fig. 8. Time series plots of seasonal counts of hot weather events (upper panels) and hot days (lower panels) observed at Les Cedres and La Tuque in the June-August summer season over the 1941-1998 period. The $T_{\min }$ threshold (u) corresponds to the 80th percentile for both locations, and the $T_{\max }$ threshold $(\mathrm{V})$ corresponds to the 95th percentile for Les Cedres and to the 85th percentile for La Tuque, obtained from the period of recorded observations. Solid lines correspond to linear time trends 

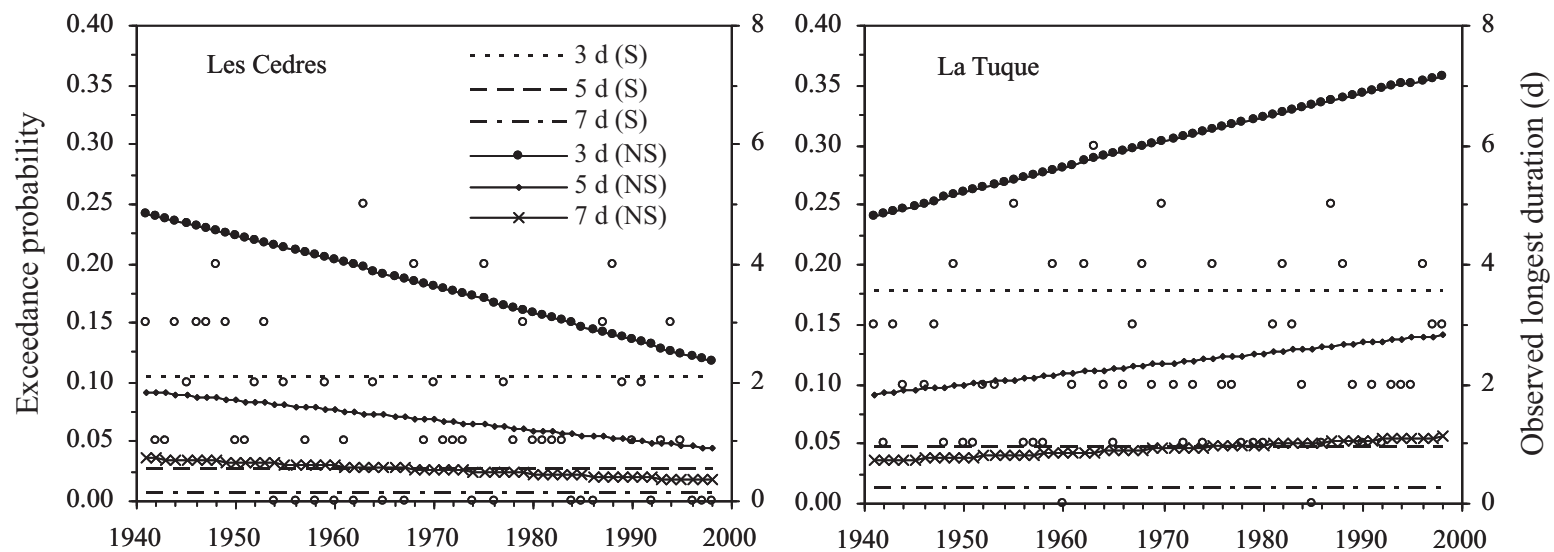

Fig. 9. Time evolution of exceedance probabilities for the 3 longest durations $(3,5$ and $7 \mathrm{~d})$ of hot weather events, obtained with stationary (S) and non-stationary (NS) models for Les Cedres and La Tuque. Observed values for the longest durations are shown with open circles (right axis)

the basis of various combinations of $T_{\min }$ and $T_{\max }$ thresholds. The usefulness of both arbitrarily chosen thresholds and those based on higher percentiles of the period of recorded $T_{\min }$ and $T_{\max }$ observations is demonstrated. Although thresholds that are arbitrarily chosen but reasonably high are useful for a single location, percentile-based thresholds may be a preferred option for regional analysis. From the various analyses presented in this study, the following conclusions and recommendations can be made:

- The CPPG model with the logarithmic distribution for durations of HWEs describes the HWE occurrence and duration processes and the number of hot days better than the CPPG model with the exponential distribution for duration of HWEs when assessed on the basis of the Kolmogorov-Smirnov and chi-squared goodness-of-fit tests. Just 3 estimated parameters, 2 for the occurrence of HWEs and 1 for their durations, not only describe adequately the 2 basic component processes, but also the distributions of the number of hot days and of the longest durations of HWEs. The

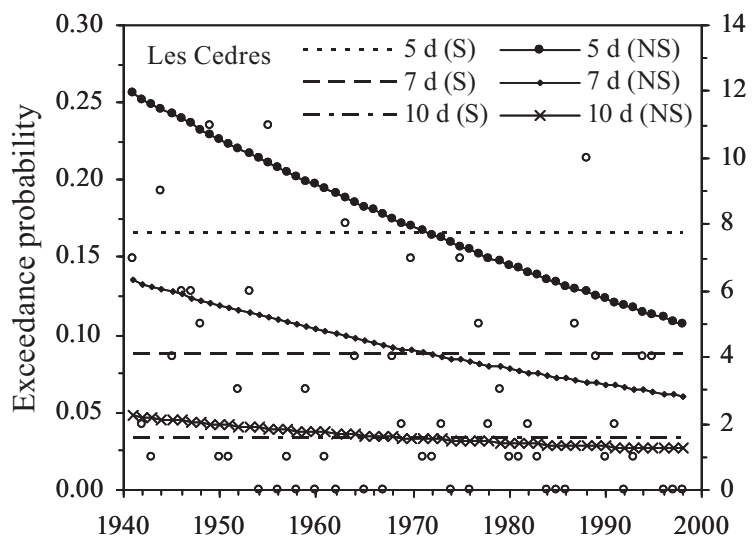

CPPG modeling framework is clearly advantageous according to the principle of parsimony.

- Various components of the hot weather climate in Montreal, on which a complete validation of the CPPG model is presented, are found to be stationary on the basis of the non-parametric Mann-Kendall and Spearman rank correlation tests. Therefore, the non-stationary framework introduced in the present study is demonstrated using HWE characteristics derived from homogenized observations of $T_{\min }$ and $T_{\max }$ recorded at 2 other locations in southern Quebec: Les Cedres and La Tuque. From a set of 5 nonstationary candidate models, the one with a linear time trend in the mean rate of occurrence of HWEs is found to be the most suitable model on the basis of AIC model selection criteria for both Les Cedres and La Tuque.

- It is anticipated that as a result of changing temperature regimes, the frequency of occurrence or severity of hot weather spells or both could change. The structure of the developed CPPG model is quite flexible, to

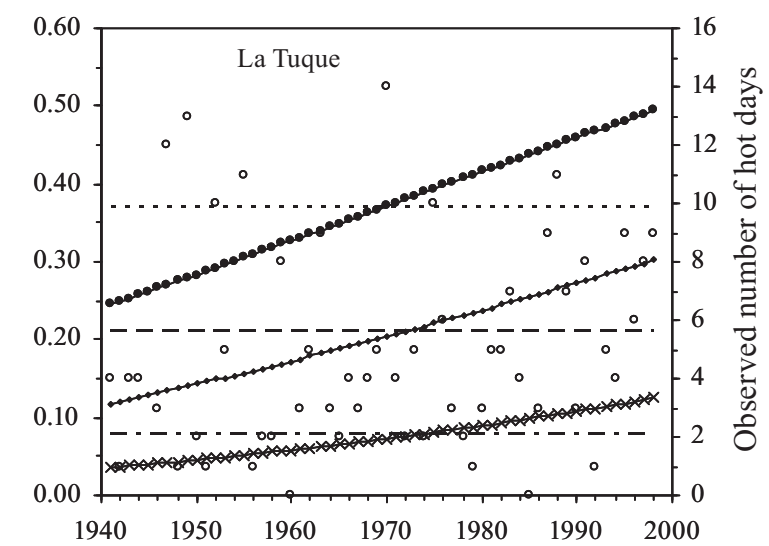

Fig. 10. Time evolution of exceedance probabilities for the 3 selected values for the number of hot days $(5,7$ and 10$)$ in the June-August summer period, obtained with stationary (S) and non-stationary (NS) models for Les Cedres and La Tuque. Observed values for the number of hot days are shown with open circles (right axis) 
accommodate both of these aspects in terms of timedependent model parameters; hence, it could be a useful tool for developing probabilistic information about the behavior of hot weather spells for a smaller or larger spatial domain.

- Occurrence of HWEs is a regional mechanism. Therefore, small homogeneous regions based on some physical factors or purely based on statistical approaches (see, for example, Hosking \& Wallis 1997) could be defined, and regional averaged time series of the 2 component processes of the CPPG model could be derived for a regional analysis. Future studies could consider this extension of the model. Furthermore, the non-stationary framework proposed is quite general. It could be extended further by including indices of large-scale atmospheric circulations in the parameters of the distributions of the number of HWEs or durations of HWEs as covariates. This will help develop non-stationary and/or non-identical frequency analysis procedures in a similar manner as for other hydrometeorological variables (see, for example, Katz et al. 2002, Khaliq et al. 2006b).

- The intensity aspect of HWEs is taken into consideration by using various thresholds with increasing magnitudes. However, if the interest lies in modeling magnitudes of exceedances, then that can be achieved by extending the univariate approach (described in Katsoulis \& Hatzianastassiou 2005 and Furrer et al. 2010) to a multivariate setting, in order to model exceedances of both daily minimum and maximum temperature thresholds. Compared to the intensity aspect, modeling seasonality of hot weather spells over the summer season is a challenging task. One possible solution would be to concentrate separately on small seasonal time windows.

Acknowledgements. The financial support provided by the Ouranos Consortium on Regional Climatology and Adaptation to Climate Change, during 2005-2006, is gratefully acknowledged. The access to Environment Canada homogenized temperature dataset is fully appreciated. We are thankful to 3 anonymous referees for their valuable comments that led to an improved article, and to Prof. André St-Hilaire for reviewing an earlier version of the paper.

\section{LITERATURE CITED}

Abaurrea J, Cebrián AC (2002) Drought analysis based on a cluster Poisson model: distribution of the most severe drought. Clim Res 22:227-235

Abramowitz M, Stegun IA (1965) Handbook of mathematical functions with formulas, graphs and mathematical tables. Dover Publications, New York

> Akaike H (1974) A new look at the statistical model identification. IEEE Trans Automat Contr 19:716-722

> Anderson RL (1942) Distribution of the serial correlation coefficient. Ann Math Stat 13:1-13
Box GEP, Jenkins GM (1970) Time series analysis: forecasting and control. Holden-Day, San Francisco, CA

Cameron AC, Trivedi PK (1998) Regression analysis of count data. Cambridge University Press, Cambridge

Cox DR, Isham V (1980) Point processes. Chapman \& Hall, London

Crutcher HL (1975) A note of the possible misuse of the Kolmogorov-Smirnov test. J Appl Meteorol 14:1600-1603

> Elsner JB, Bossak BH (2001) Bayesian analysis of US hurricane climate. J Clim 14:4341-4350

Feller RW (1968) An introduction to probability theory and its applications, Vol 1. John Wiley, New York

Furrer EM, Katz RW, Walter MD, Furrer R (2010) Statistical modeling of hot spells and heat waves. Clim Res 43: 191-205

Guttorp P (1995) Stochastic modeling of scientific data. Chapman \& Hall, London

Hosking JRM, Wallis JR (1997) Regional frequency analysis. Cambridge University Press, Cambridge

IPCC (Intergovernmental Panel on Climate Change) (2002) IPCC workshop report on 'changes in extreme weather and climate events', Beijing, China, 11-13 June, 2002. Available at www.ipcc.ch/publications_and_data/publications_and_ data_supporting_material.shtml (accessed on 14 September 2010)

Johnson NL, Kotz S, Kemp AW (1992) Univariate discrete distributions. John Wiley, New York

Katsoulis BD, Hatzianastassiou N (2005) Analysis of hot spell characteristics in the Greek region. Clim Res 28:229-241

Katz RW (2002) Stochastic modeling of hurricane damage. J Appl Meteorol 41:754-762

Katz RW, Parlange MB, Naveau P (2002) Statistics of extremes in hydrology. Adv Water Resour 25:1287-1304

Kendall MG (1975) Rank correlation methods. Charless Griffin, London

Kendall MG, Stuart A (1977) The advanced theory of statistics, Vol 1. Charless Griffin \& Company, London

> Khaliq MN, St-Holaire A, Ouarda TBMJ, Bobée B (2005) Frequency analysis and temporal pattern of occurrences of southern Quebec heatwaves. Int J Climatol 25:485-504

Khaliq MN, Gachon P, St-Hilaire A, Ouarda TBMJ, Bobée B (2006a) Southern Quebec (Canada) summer-season heat spells over the 1941-2000 period: an assessment of observed changes. Theor Appl Climatol 88:83-101

- Khaliq MN, Ouarda TBMJ, Ondo JC, Gachon P, Bobée B (2006b) Frequency analysis of a sequence of dependent and/or non-stationary hydro-meteorological observations: a review. J Hydrol (Amst) 329:534-552

Khaliq MN, Ouarda TBMJ, St-Hilaire A, Gachon P (2007) Bayesian change-point analysis of heatspell occurrences in Montreal, Canada. Int J Climatol 27:807-818

Medhi J (2002) Stochastic processes. New Age International, New Delhi

Parzen E (1964) Stochastic processes. Holden-Day, San Francisco, CA

> Prieto L, Herrera RG, Díaz J, Hernández E, del Teso T (2004) Minimum extreme temperatures over Peninsular Spain. Global Planet Change 44:59-71

Reiss RD, Thomas M (2007) Statistical analysis of extreme values. Birkhäuser Verlag, Basel

> Rodriguez-Iturbe I, Cox DR, Isham V (1987) Some models for rainfall based on stochastic point processes. Proc R Soc Lond A 410:269-288

Rootzén H, Tajvidi N (1997) Extreme value statistics and wind storm losses: a case study. Scand Actuar J 1997:70-94

Skinner CJ (1992) On identification disclosure and prediction disclosure for micro data. Stat Neerl 46:21-32 
Snedecor G, Cochran WG (1980) Statistical methods. The Iowa State University Press, Ames

Strupczewski WG, Singh VP, Mitosek HT (2001) Nonstationary approach to at-site flood frequency modeling. III. Flood analysis of Polish rivers. J Hydrol (Amst) 248: 152-167

Vincent LA, Zhang X, Bonsal BR, Hogg WD (2000) Homogenized daily temperatures for trend analyses in extremes over Canada. In: Preprint 12th Conf Applied Climatology. Ashville, NC, AM Meteorol Soc p 86-89
Vincent LA, Zhang X, Bonsal BR, Hogg WD (2002) Homogenization of daily temperatures over Canada. J Clim 15: 1322-1334

Von Storch H, Zwiers FW (1999) Statistical analysis in climate research. Cambridge University Press, Cambridge

Walpole RE, Myers RH (1989) Probability and statistics for engineers and scientists. Macmillan Publishing Company, New York

Zelenhasić E, Salvai A (1987) A method of streamflow drought analysis. Water Resour Res 23:156-168

Appendix 1. Distribution function of the number of hot days for the compound Poisson process model

If $X$ is assumed to follow the discrete logarithmic distribution then the conditional probability $P(M=m \mid N=n)$ can be derived from the probability generating function (pgf) of $X$, which is given by Kendall \& Stuart (1977, p. 139) as:

$$
G(s)=E\left[s^{X}\right]=\frac{\log (1-b s)}{\log a}
$$

Given $N=n$, the conditional distribution of $M$ is the sum of $n$ independent random variables. The pgf of $M$ is the $n$-fold convolution of $G(s)$, i.e.:

$$
[G(s)]^{n}=\frac{1}{(\log a)^{n}}[\log (1-b s)]^{n}
$$

Using the standard theorems of combinatorial mathematics (Abramowitz \& Stegun 1965, p. 824), the second term on the right-hand side of Eq. (A2) can be transformed in terms of Stirling's numbers of first kind, i.e. the pgf of $M$ can be written as:

$$
[G(s)]^{n}=\frac{n !}{(\log a)^{n}} \sum_{j=n}^{\infty}(-1)^{j} S N F_{j}^{n} \frac{(b s)^{j}}{j !}
$$

where $S N F_{j}^{n}$ is the Stirling's number of the first kind. In the expansion of the pgf, Eq. (A3), as a power series in $S$, the $P(M=m \mid N=n)$ is given by the coefficient of $s^{j}$ (see Medhi 2002, p. 7). Thus:

$$
P(M=m \mid N=n)=(-1)^{m} \frac{b^{m} n !}{(\log a)^{n} m !} S N F_{m}^{n}
$$

After some algebraic manipulations, the unconditional probability, $P(M=n)$, in the case of the basic compound Poisson process, i.e. when Eq. (1) is applicable, and in the case of the CPPG model can be derived from Eq. (A4). Corresponding expressions are provided in Eqs. (9) and (10), respectively, in the main article. 